\title{
Trivium
}

Revue franco-allemande de sciences humaines et sociales - Deutsch-französische Zeitschrift für Geistesund Sozialwissenschaften

1 | 2008

"Iconic Turn » et réflexion sociétale

\section{Les images, acteurs majeurs de la connaissance}

À propos de la poiesis et de l'episteme des images langagières et visuelles

\section{Sigrid Weigel}

Traducteur : Denis Trierweiler

\section{OpenEdition}

\section{Journals}

Édition électronique

URL : http://journals.openedition.org/trivium/319

DOI : 10.4000/trivium.319

ISSN : 1963-1820

Éditeur

Les éditions de la Maison des sciences de l'Homme

\section{Référence électronique}

Sigrid Weigel, "Les images, acteurs majeurs de la connaissance », Trivium [En ligne], 1 | 2008, mis en ligne le 08 avril 2008, consulté le 08 septembre 2020. URL : http://journals.openedition.org/trivium/ 319 ; DOI : https://doi.org/10.4000/trivium.319

Ce document a été généré automatiquement le 8 septembre 2020.

\section{c) (7) $\odot$}

Les contenus des la revue Trivium sont mis à disposition selon les termes de la Licence Creative Commons Attribution - Pas d'Utilisation Commerciale - Pas de Modification 4.0 International. 


\section{Les images, acteurs majeurs de la connaissance}

À propos de la poiesis et de l'episteme des images langagières et visuelles ${ }^{1}$

\section{Sigrid Weigel}

Traduction : Denis Trierweiler

\section{NOTE DE L'ÉDITEUR}

Nous tenons à remercier chaleureusement Sigrid Weigel ainsi que les éditeurs de la publication originale de nous avoir accordé le droit de traduire son article.

\section{Un déluge d'images}

1 De toute part, des plaintes sur l'avalanche d'images qui nous assaille sont audibles. De sombres pronostics annoncent même la fin proche de la littérature, si ce n'est de

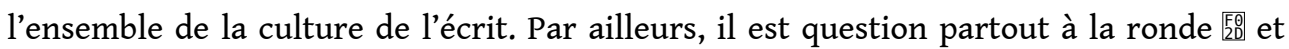
particulièrement dans les sciences ${ }_{20}^{6}$, d'un iconic ou pictorial turn qui a nourri des aspirations à une nouvelle science interdisciplinaire de l'image. Mais simultanément est aussi conjurée une fin de l'image, parce qu'aujourd'hui les artistes ne s'expriment plus guère à travers le médium traditionnel de la forme imagée, de la peinture, mais par des installations, des objets, des actions et des vidéos - ce dont tout un chacun peut se convaincre par une visite à la Biennale ou à la Documenta.

Qu'en est-il donc de l'image et dans quelle mesure la situation actuelle se distingue-telle de circonstances plus anciennes?

2 Il semble bien que notre quotidien soit imprégné d'images et réglé par elles. Nous sommes habitués à nous orienter selon des images dans la vie de tous les jours : des panneaux de stop et de priorité, de petits bonshommes marchants ou arrêtés, des indications d'entrée, d'issues de secours et de places de stationnement, des 
pictogrammes d'avions, de bus et de trains. Pourtant, de telles indications ou signaux, tableaux indicateurs ou d'interdiction n'ont que peu de rapport avec ce que l'on associe couramment au terme d'image. De fait, il s'agit plutôt de symboles du répertoire d'un code du quotidien dans lequel des représentations graphiques endossent exactement le même rôle que, par exemple, des chiffres, des lettres, des couleurs ou des sons. Ils nous aident à nous orienter - que ce soit sous la forme de numéros de domiciles, de feux de circulation ou de signaux téléphoniques. L'énorme augmentation de tels codes est liée au développement de l'infrastructure, elle n'a cessé de s'accélérer depuis l'époque postale. À l'âge de la communication électronique et du world wide web, le développement de ce genre de symboles a fait un bond considérable, car ils sont compréhensibles internationalement, c'est-à-dire de manière globale, par-delà tout problème de traduction au sein d'un monde de confusion babylonienne des langues.

Pourtant ce développement n'a, à vrai dire, rien à voir avec un déluge d'images ; on pourrait tout au plus parler d'un refoulement partiel de l'alphabet par des symboles graphiques et des diagrammes. Ce qui signifie que dans la communication quotidienne, la langue se trouve de plus en plus remplacée par un système de codes internationalement valable. En outre, les nombreux graphiques diffusés tous les jours dans les journaux et à la télévision concernant les informations boursières (comme par exemple la courbe du cours des actions en bourse sur une durée d'un mois) montrent que l'expression "déluge d'images" masque le fait que notre culture devrait bien plutôt être décrite comme une époque dominée par des données.

Mais revenons aux images.

4 Les informations sur les événements du monde sont, elles aussi, transmises et perçues avant tout à travers des images, bien que, comme on l'a constaté depuis longtemps, seules des images isolées ou très souvent répétées (comme celles des avions entrant en collision avec les tours jumelles de New York) s'imprègnent dans la conscience, tandis que les images informatives que nous voyons quotidiennement à la télévision se nivellent réciproquement par leur succession rythmique rapide. Le cumul des images d'information tend à effacer les images précédentes avant que celles-ci n'aient pu s'imprégner.

Plus il y a d'images, plus leur enchaînement est rapide, et moins elles ont d'effet. La validité de cette proposition est cependant limitée : elle ne vaut que jusqu'au point où nos yeux perçoivent consciemment les images. Si le rythme temporel de l'œil est pris de court par des images trop brèves, celles-ci s'inscrivent manifestement dans notre mémoire sans que nous en soyons conscients. Plutôt que de parler d'un déluge d'images, il serait donc plus juste de parler du rythme des images. ${ }^{2}$ Ainsi, on peut situer une césure radicale dans l'histoire de la perception au moment précis où les images ont appris à courir. C'est l'invention du film qui a provoqué un choc dans la conscience humaine, par la "dynamite de ses dixièmes de seconde ", comme l'a formulé Walter Benjamin ${ }^{3}$ dans les années 1930. Pourtant, de nos jours, ses observations ne suscitent plus qu'un sourire bienveillant de notre part, les images en mouvement n'étant pas seulement devenues entre-temps une habitude dans nos salons et pour notre œil; comparées à la cadence qu'exigent de notre attention les moyens de communication électroniques, elles compteraient davantage parmi les formes de perception visuelle plutôt reposantes. Par conséquent, à y regarder de plus près, la formule du déluge des images se révèle être plutôt une critique culturelle de la société médiatique, de la domination des mass médias. Elle a bien sûr été, depuis longtemps, absorbée par les 
médias eux-mêmes, puisque la critique télévisuelle s'est octroyée sa place attitrée dans les journaux et les magazines de télévision. Lorsqu'il s'agit, en revanche, du refoulement par les images de la culture de Gutenberg, c'est-à-dire d'une relation modifiée entre textes et représentations visuelles, alors il est permis de douter du fait que les images aient vraiment pris une importance tellement plus grande dans notre culture qu'en des temps antérieurs. Car avant la faculté de lire, qui ne s'est mise à concerner des parts importantes de la population qu'aux alentours de 1800 (à travers les programmes d'alphabétisation), les impressions des feuilles volantes n'étaient pas moins importantes que les textes des chanteurs ambulants qui les accompagnaient. ${ }^{4}$ Quant au journal, il n'est, lui aussi, devenu peu à peu un média de masse qu'avec le progrès des possibilités techniques de reproduction d'images imprimées, au XIX siècle. Quoi qu'il en soit, dans l'histoire de la culture européenne, les phases au cours desquelles texte et image agissent de concert sont plus nombreuses que celles où elles sont en concurrence.

\section{Iconic Turn}

6 Mais qu'en est-il du malaise évoqué quant à la communication visuelle envers cette orientation proprement obsessionnelle qui est liée à la formule de l'iconic turn? Comment la critique de l'image s'accommode-t-elle de la passion pour les images, à laquelle répondent des volumes aussi chèrement conçus que, par exemple, le Bilderwissen [Savoir des images] ${ }^{5}$ de B. Martin Kemp, ou les nombreux projets d'exposition et de manifestation dans lesquels, aujourd'hui, l'art et la science se tendent la main ?

7 Ce sont avant tout les possibilités des présentations générées par ordinateur et les nouveaux procédés imagés, dont se servent les sciences techniques et les sciences de la nature, qui ont déclenché l'iconic turn : il s'agit là, par exemple, des visualisations dans le domaine de la physique des particules, qui, par des projections spécifiques, permettent de mettre en évidence des corrélations entre particules ${ }^{7}$, des représentations de champs vectoriels en mathématique ${ }^{8}$, ou des représentations colorées de valeurs d'un radio spectromètre mesurant l'intensité radio sur la base de signaux solaires ${ }^{9}$.Un autre exemple sont les cartes du cerveau répandues dans les mass médias - ces prises de vue de couleur turquoise criante des tomographies par émission de positrons (TEP) dans lesquelles les régions actives du cerveau des patients sont rendues visibles par injection de tissus émetteurs actifs, qui agissent en tant que marqueurs des zones du cerveau actives en changement de matière, ou bien ces cartes grises du cerveau de l'imagerie par résonance magnétique fonctionnelle (IRMf), dans lesquelles les régions actives sont marquées de couleur jaune, et qui sont la traduction visuelle de données (valeurs de mesure du flux sanguin et de l'activité de l'oxygène selon la différence entre état normal et activité, qui sont représentées par des valeurs colorées différentes).

8 Ces nouveaux procédés imageants agissent comme des voyages d'exploration en des terres qui étaient jusque-là soustraites à la visibilité. Les observe-t-on comme des images, la première chose qui frappe le regard est la beauté des représentations. Beauté qui atteste à quel point ces procédés techniques sont eux aussi marqués du sceau des habitudes visuelles qui ont été acquises par l'observation de l'art. Mais leur facture esthétique masque le fait qu'il ne s'agit pas là d'images au sens conventionnel, mais 
essentiellement de procédés de mesure et de visualisation de données. Car ce que réalisent les procédés imageants se trouve par-delà la copie et la représentation, pardelà la mimésis et l'analogie. Les procédés imageants engendrent des présentations [Darstellungen] auxquelles ne revient aucun caractère iconique ou pictural. Ils ne présentent pas des choses ou des faits, mais des fonctions, des activités, des spécificités ou des états de faits qui ont été consignés à l'aide d'indicateurs précis. Ils recèlent malgré tout une énorme force suggestive, comme si grâce à eux l'intérieur ou les secrets de la nature se présentaient directement sous nos yeux. Et cet effetest fondé sur le fait qu'ils agissent comme des images et, du coup, participent aussi à la prétention de vérité des images. Ainsi vu, ainsi cru.

Dans cette mesure, parler d'iconic turn induit en erreur si l'on cherche à affirmer par là un tournant vers l'image iconique ou picturale, car derrière cette formule se cache un développement qui devrait en fait être décrit comme un tournant vers une culture visuelle par-delà les images. Il n'est pas douteux que, dans la culture électronique, les médias traditionnels que sont l'écrit, l'image et le nombre se rencontrent en un seul et unique point de fuite : dans le système de rédaction digital. Et pourtant, il y a bien un pictorial turn, un tournement vers les images. Car - et c'est ma thèse - qu'il s'agisse des graphiques multicolores des structures moléculaires et de la double hélice, ou des images colorées du cerveau, ou encore des représentations de l'univers générées par ordinateur - cette pléthore d'images techniques dont nous sommes entourés a réveillé une nostalgie pour les vieilles images. Au milieu des images bigarrées sur papier glacé, par lesquelles les gènes et le cerveau sont devenus de véritables icônes des médias, est né un désir d'images graphiques et de photographies provenant des chambres aux merveilles du passé, un désir de représentations venu d'une époque à laquelle l'imagination avait encore son mot à dire dans les images que nous nous faisions de la nature et de ses lois. ${ }^{10} \mathrm{En}$ ce sens, la formule de l'iconic turn peut se comprendre comme un symptôme qui témoigne d'une renaissance, et parfois aussi d'un abandon nostalgique à des images d'une époque à laquelle le royaume du visible était encore sévèrement séparé des sphères mystérieuses de la création, et à laquelle cette ligne de démarcation devait être surmontée à l'aide de la fantaisie iconographique. Cette tentative de superposition de l'invisible par du visible n'aura sans doute jamais été plus perceptible dans une image de l'histoire de la science, que dans le crâne colorié par Franz Gall ${ }^{11}$, dont les lignes et les écritures sur la surface révèlent l'effort pour représenter l'«intérieur de l'esprit» à l'«extérieur du crâne ».12 C'est tout particulièrement dans la transmission des connaissances populaires acquises en médecine et dans les sciences de la nature - comme ambitionne de le faire de façon exemplaire l'ouvrage en plusieurs volumes de Fritz Kahn sur la vie de l'homme, Das Leben des Menschen ${ }^{13}$ - que l'on trouve des planches où des fonctions physiologiques sont traduites en scénarios iconiques, comme dans le tableau de Kahn sur la fonction de l'excitation motrice ${ }^{14}$, qui montre une coupe transversale d'un buste humain sur lequel apparaît, dans le crâne ouvert, l'atelier d'un "centre moteur " qui est relié par des conduits aussi bien à l'intérieur du corps qu'au monde extérieur; de sorte que la métaphore de la conduite apparaît ici littéralement comme l'image qui « relie le monde avec les figures du savoir $» .^{15}$

10 Cette illustration est particulièrement parlante : elle représente l'action conjointe de la fonction cérébrale et du mouvement moteur, la relation entre l'activité d'une région donnée du cerveau et la célèbre pression sur le bouton. Il surmonte, grâce à l'image du conduit, la black box de nos connaissances sur la relation spécifique entre l'activité 
motrice et les processus neuronaux invisibles qui l'accompagnent: le conduit électrique comme métaphore du fonctionnement des neurones qui ne sont pas, euxmêmes, visibles. La métaphore vient donc prendre la place des points aveugles dans le savoir. Sa fonction est double, en ce qu'elle veille simultanément à marquer et à cacher l'inconnu ou le provisoire dans les explications sur les lois naturelles. C'est pourquoi, dans le commerce ultérieur avec de telles images, il importera de s'assurer comment l'on procède avec ce double statut de la métaphore du conduit.

11 Pendant des siècles, notre désir d'en savoir plus sur la manière dont nous pensons et dont nous ressentons, la manière dont nos représentations et notre langage se forment dans la tête, a été lié au fantasme de pouvoir regarder à l'intérieur de la tête. "Nous devrions nous fracturer la boîte crânienne et en retirer nos pensées en même temps que les fibres cérébrales ", comme le faisait dire Büchner, voici un siècle et demi, à son Danton de fiction s'adressant à sa bien-aimée Julie. Ce que le psychologue Wilhelm Wundt qualifiait encore, vers 1900, de "fantasme du futur", à savoir "qu'un jour viendra où notre connaissance de la mécanique cérébrale sera bien plus complète $»^{16}$ semble aujourd'hui être devenu réalité. La médecine est effectivement capable aujourd'hui de littéralement scruter le cerveau humain. Certes, les images qu'elle trouve ne sont pas les représentations, les fantaisies et les rêves des hommes; ce sont plutôt, comme nous l'avons montré, les présentations d'activités neuronales, que l'on appelle des cartes, et qui décrivent et documentent des états précis de processus biochimiques. Ainsi, grâce aux procédés imageants, nous disposons aujourd'hui d'images du cerveau, mais nous ne savons toujours pas comment fonctionnent la pensée et le sentiment. Et surtout, nous ignorons comment les images naissent dans la tête, et de quelle manière sont engendrées des significations qui se lient à des "mouvements d'humeur» spécifiques. ${ }^{17}$ En un mot: nous disposons aujourd'hui d'images de la pensée, mais nous savons peu de choses sur ce que les images signifient pour la pensée.

\section{Penser avec des images}

12 Si je pars du fait que les images jouent un rôle central dans nos connaissances, dans notre savoir et dans nos affects, c'est bien sûr à la condition de présupposer que le concept d'image englobe davantage et autre chose que les images iconiques et techniques, ou même l'ensemble du champ des représentations visuelles. Car les archives virtuelles d'images sont bien plus vastes que celles que l'on peut voir sur les murs et sur les tableaux, sur les écrans de télévision et dans les livres, dans les musées, dans les métros et sur les écrans de cinéma. Car ce que l'on appelle les images dans la tête précèdent leur présentation matérielle ou médiale, ce que le langage démontre très bien: lorsque nous disons par exemple que nous nous faisons une image, ou lorsqu'il est question de représentations. Ce qui est en jeu c'est la question de savoir comment naissent des significations spécifiques et quel est le rôle qu'y jouent les divers sens et les perceptions concrètes, le rôle qu'ont la mémoire et le langage. Il s'agit de savoir comment des perceptions et des expériences immédiates se lient à des images du souvenir.

13 Les images, en ce sens étendu et plus fondamental, sont des condensations dans lesquelles se rejoignent des impressions passées et actuelles et se compriment en une apparence donnée, ou, pour le dire avec les mots de Walter Benjamin : «Une image [...] 
est ce en quoi l'Autrefois rencontre le Maintenant dans un éclair pour former une constellation. $»^{18}$ Les images sont les acteurs principaux de notre pensée, parce qu'elles accomplissent cette réalisation prodigieuse qui consiste à relier des choses et des événements du monde extérieur, ou bien des vécus personnels, avec les archives de notre savoir. La signification littérale des termes «image » [Bild] et « former » [bilden] renvoie à ce processus par lequel sont générées des représentations, en ce que, tout d'abord elles se forment, prennent une apparence [Gestalt] et, pour ce faire, ont recours au répertoire du langage disponible, aux signes et aux symboles qui sont engrangés dans la mémoire culturelle et individuelle. C'est pourquoi le théoricien de l'image américain, W. J. T. Mitchell, dit : "L'image est le concept général, ramifié en diverses analogies et correspondances (convenientia, aemulatio, analogia, sympathia), qui maintient la cohésion du monde par des images du savoir. $»^{19}$ L'image, donc, comme agrafe entre le monde et le savoir.

Car, à l'encontre d'un préjugé persistant, la pensée n'est en aucune façon abstraite. Là encore, la littéralité nous conduit à une piste intéressante. Le mot «théorie » nous rappelle que la connaissance désigne tout d'abord la contemplation et l'intuition, car le grec theoria désigne à l'origine celui qui voit un spectacle. Il remonte à la tradition des communautés festives sacrales, à partir desquelles est né, par la suite, le théâtre. Et si, depuis Platon, "théorie » est devenu le terme désignant une connaissance pratiquée systématiquement, c'est que la pensée est née d'une contemplation organisée sous forme de rite. C'est seulement au cours du développement de l'histoire européenne que s'est perdue la conscience que la pensée est née d'une scène de contemplation, tandis que simultanément les images devinrent de simples objets de contemplation artistique, perdant ainsi largement leur rôle actif.

Par conséquent, penser avec des images et en images peut parfaitement signifier aujourd'hui de reconquérir une culture qui pénètre sur la scène de la connaissance. Cependant, ce qui déterminera sa réussite c'est de savoir si elle considère cette scène comme un supermarché d'images toutes faites, ou bien plutôt comme un atelier de la connaissance. Les médias et le progrès des instruments et des procédés techniques ne sont en aucune façon exclus de cette démarche - à condition pourtant que leur utilisation obéisse au slogan qu'avait déjà formulé Fontenelle dans son Dialogue des morts de 1683 : les yeux qui ont besoin de l'aide de nouvelles lunettes sont ceux des philosophes, c'est-à-dire ceux de tout homme, quel que soit son activité, en tant que philosophe.

Niklas Luhmann a revendiqué, une fois, qu'il existât une sorte de poésie parallèle pour des réalisations théoriques ambitieuses. ${ }^{20} \mathrm{Je}$ voudrais réfuter cela : ce qui m'importe n'est pas un côte à côte entre théorie et poésie, mais la scène de la connaissance ellemême, sur laquelle les images visuelles et langagières jouent les rôles principaux.

\section{Petite histoire de l'image matérielle}

Si le concept de l'image n'est le plus souvent lié qu'à des images matérielles, à des copies ou à des représentations visuelles, il faut voir là le résultat d'une réduction de l'image à la toile ou au tableau bidimensionnel au cours de l'histoire de la culture européenne. Alors qu'à vrai dire, dans la tradition occidentale, l'image est née du culte, d'une constellation que George Didi-Huberman a décrite comme Devant l'image. ${ }^{21}$ L'historien des images Hans Belting a, lui aussi, montré, dans Image et culte. Une histoire 
de l'art avant l'époque de l'art de $1990^{22}$, combien la tradition imagée iconographique était étroitement liée au culte des morts. Les portraits et les représentations des morts sur les pierres tombales ou les lieux de culte, que nous ont transmis les cultures antiques et le premier christianisme, attestent que des présentations imagées sont apparues en tant que remplaçants des morts. C'est ainsi que l'Imago, c'est-à-dire le portrait personnel, devenu surtout dans la culture chrétienne l'objet privilégié des pratiques religieuses, a marqué l'histoire de l'imagination. Depuis lors, la faculté imaginative est peuplée de remplaçants de personnes absentes ou mortes.

L'origine des images étant ce seuil entre la vie et la mort, on comprend que les représentations de corps humains soient souvent affectées d'une inquiétante étrangeté. C'est la raison pour laquelle les portraits colorés de corps depuis longtemps décomposés apparaissent souvent comme des revenants du royaume des morts. C'est à cela que fait par exemple allusion Heinrich Heine, lorsque, au cours de son Voyage de Munich à Gènes (1828), il observe de vieux tableaux au Palais Durazzo, parmi lesquels un tableau de Giorgione qu'il qualifie de "frappant par son silence " [totschweigend getroffen], et qu'il formule, à la vue de tableaux de Cornelius et de Rubens, son impression que les figures représentées semblent toutes porter en elles un germe de mort. $^{23}$

La présentation imagée de personnes mortes signifie la présence des absents in effigie. Dans cette même tradition s'inscrivent aussi les portraits de souverains, et surtout les sculptures de souverains. En tant que copies du corps du roi mortel, il leur incombait après sa mort de représenter le corps symbolique du roi, jusqu'à ce que le nouveau roi soit investi et intronisé, afin d'assurer la continuité de la royauté durant cette période intérimaire. ${ }^{24}$ Les descendants de ces corps de souverains tridimensionnels in effigie peuplent aujourd'hui encore, sous forme de monuments, notre paysage urbain - même si presque plus personne ne daigne les gratifier d'un regard. Déjà Robert Musil avait pu constater qu'il n'y avait rien de plus invisible que les monuments, dont la caractéristique la plus remarquable était qu'on ne les remarquait pas. ${ }^{25}$ Les monuments ne sont pas seulement des images iconiques transformées en pierres, mais ils incarnent très exactement la tendance de l'iconographie à la pétrification et à une perte d'efficience qui croît avec le temps.

Mais l'intrication de l'image et du culte est aussi la raison qui fait que l'histoire des images s'accompagne constamment d'une oscillation entre hostilité aux images et vénération. L'iconoclasme, la destruction des images, appartient tout autant à notre histoire que l'idolâtrie, l'adoration des images. Ainsi n'est-il pas rare que des conflits de croyance se vident se livrent sous la forme de combats d'images, comme l'a montré à nouveau tout récemment la destruction des statues de Bouddha par les talibans. D'un autre coté, le moment cultuel de la représentation personnelle a déployé des effets tout à fait nouveaux et spécifiques, par-delà la religion justement, dans le monde des médias modernes. Il est aujourd'hui au centre de la culture pop, qui se présente comme un répertoire d'icônes modernes, avec, au sommet, la star portant le nom symbolique de Madonna.

21 Moins connu, parce que moins spectaculaire, est le fait que notre histoire n'eut pas seulement à consigner des destructions répétées d'images, mais qu'elle est aussi fortement marquée par une hostilité aux lettres. Le reproche d'obéissance à la lettre est l'expression d'une hostilité à l'écrit ${ }^{26}$, qui a souvent été dirigée contre des érudits, parfois contre des juristes, et régulièrement, directement ou indirectement, contre la 
tradition juive. Elle ne discrimine pas l'écrit ou le texte en soi, mais l'art de l'explication et de la lecture, elle se dirige contre cette pratique de l'exactitude et du détail qui est parfois capable de découvrir dans un petit signe, un accent ou une nuance davantage de signification que dans une phrase entière.

Et cette hostilité à l'encontre du détail ${ }^{27}$ n'est pas seulement réservée aux textes, elle émerge aussi dans l'histoire des images visuelles. Par exemple, lorsque l'on traitait la photographie, juste après son invention, avec méfiance et rejet, avec cet argument que, en ce qu'elle montrait tous les détails, même ceux invisibles à l'œil nu - comme les rides dans la peau du visage et les plis dans la veste des portraits des "grands hommes »-, elle serait trop " fidèle à la lettre ${ }^{28}$ Ce qui devint le motif pour l'exclure du royaume de l'art, car la destination de l'art était de représenter le contexte, le grand tout.

Cette identification de l'art et de la vue d'ensemble est le résultat d'une soumission des images au cadre des tableaux et des peintures dans l'histoire de l'art européenne. L'isolation de sculptures et de peintures de leur contexte cultuel, puis leur dépôt dans des musées, galeries et expositions est la condition de possibilité de cette forme de contemplation de l'art qui a forgé le concept d'image aujourd'hui dominant. Ce développement est lié à des modifications radicales des habitudes de voir et de l'attitude qu'adopte l'individu vis-à-vis de l'image. Car, dans le culte, l'image et la personne sont partie intégrante d'une seule et même scène. L'observateur s'y trouve pour ainsi dire dans l'image. On voit encore des retentissements épars de cette tradition au XVIII siècle, par exemple lorsque Wilhelm Heinse, dans ses «Lettres de Düsseldorf sur des tableaux » [Düsseldorfer Gemäldebriefe] (1776-77), décrit le tableau de Rubens La bataille des Amazones, en faisant débuter la description non pas à gauche, comme nous y sommes habitués, mais en haut à droite de l'espace du tableau - en désignant toutefois ce côté comme étant le gauche : «Le commencement, à gauche du tableau, décrit en une mêlée déjà lointaine la fuite des femmes et des chevaux. Pardessus, quelques chevaux de combat, débarrassés de leurs cavaliers, quittent le pont. $»^{29}$ C'est là un mode de description de l'image par lequel l'auteur lui-même se place dans la mêlée de la bataille. Ce n'est qu'avec l'accrochage sériel et systématique d'images dans les musées que s'est développée la convention de la vue plongeante, qui présuppose et promet un survol à distance de la totalité de l'image. Dans cette mesure, la sécularisation des images est l'une des conditions de possibilité centrales de la genèse du mode du voir propre à l'histoire de l'art. ${ }^{30}$

À l'heure actuelle, de nombreux artistes travaillent à l'encontre de cette tendance à la vue plongeante et globale. Par leurs « sortie de l'image ", ils sont littéralement sortis du cadre bidimensionnel. Dans leurs projets, comme par exemple les installations spatiales de Dan Graham ${ }^{31}$, ou les objets de Bruce Nauman dans lesquels on peut entrer ${ }^{32}$, il s'agit fréquemment et au sens le plus littéral, de rendre l'art à nouveau pénétrableet accessible d'une autre manière: à savoir par le corps. Ils actualisent par là une ancienne tradition, dans laquelle l'observateur se trouvait dans l'espace de l'image. Dans le contexte des tentatives de reconquérir une culture qui pénètre sur la scène de la connaissance, cette scène est ici matérialisée et incarnée par l'espace de l'image.

Eu égard à l'observation des images, les possibilités de reproductibilité technique n'ont pas entraîné la révolution qui a souvent été évoquée à leur propos. Elles n'ont pas établi de nouvelles conventions mais n'ont fait que renforcer la tendance à la vue plongeante, avec cet effet que, aujourd'hui, même de gigantesques images murales, comme la 
fresque de Leonard de Vinci, Ultima Cena, à Milan, ou bien la peinture surdimensionnée de Picasso, Guernica, à Madrid, dont on ne pouvait guère avoir une vue d'ensemble sur les lieux, sont facilement accessibles sous forme de cartes postales. Dès lors, dans les archives digitales de la culture du CD, l'histoire de l'art canonisée devient disponible comme un tout, par exemple avec ces 5555 chef-d'œuvres qui sont vendus sous forme d'archives digitales dans un coffret de $10 \mathrm{CD} \cdot{ }^{33}$ Dans ce format, les images ont rétréci à la taille d'un timbre poste, lorsqu'on les appelle par cette fonction, qui réunit chaque fois quinze images en vue globale. Par le stockage électronique, les conventions perceptives établies de la vue plongeante et du survol n'ont donc été que renforcées. En revanche, les spécificités des images iconiques se sont radicalement modifiées avec la technique digitale. Avec les possibilités de transformation et de manipulation des images digitales, même le médium technique de la photographie a complètement changé de visage. C'est ce que montre une comparaison entre des images de l'époque des pionniers de l'histoire de la photographie, par exemple la scène prise dans les années 1840 par David Octavius Hill dans un cimetière - auquel Walter Benjamin a rendu hommage dans sa Petite histoire de la photographie, comme lieu idéal de villégiature pour ces " premiers hommes reproduits » disposés à la durée ${ }^{34}-$ avec des exemples puisés dans les archives de photos digitales, tels les Fictitious Portraits de Keith Cottingham. ${ }^{35}$ Et une fois de plus, c'est avant tout sur l'Imago, la représentation personnelle, que sont expérimentées des techniques de transformation et des procédés de perfectionnement. Avec les Fictitious Portraits, la facultas fingendi est entrée dans une nouvelle ère. Car, avec la technique des images digitales, il semble que l'imagination soit congédiée de son rôle en tant que langage singulier dans le royaume du virtuel, puisque de nos jours, les fictions sont transformées en représentations virtuelles. Étant donné qu'au commencement de l'image matérielle se trouvait la naissance de la copie à partir du culte des morts, ce n'est sans doute pas un hasard si la fin de l'image iconique est liée à des photographies digitales dans lesquelles apparaissent des fantasmes de corps immortels.

\section{Texte et image : iconicité de l'écriture et images linguistiques}

Souvent la concurrence entre le texte et l'image s'exerce comme combat pour le droit de primogéniture, dans laquelle les adeptes $d u$ " $\mathrm{Au}$ commencement était le verbe » font irréconciliablement face aux adeptes du « Au commencement était l'image ». Mais, comme toutes les questions d'origine, cette lutte pour la position originelle est vaine. Dans le meilleur des cas, on pourrait dire : Au commencement était la trace. Car les traces précèdent les images et les signes - et ce, aussi bien dans une perspective historique que, eu égard à la production de représentations isolées présentant une signification codée. C'est ainsi que les peintures archaïques de la grotte de Lascaux ${ }^{36}$ précèdent les fresques murales antiques tout autant que l'empreinte précède la sculpture. ${ }^{37}$ Et les images iconiques doivent également leur naissance au fait qu'en elles, ces traces qui les précèdent sont recouvertes. Ce n'est que par la transformation de ces dernières en une copie analogue au corps que la soi-disant image vraie, la Vera Icon, a $\mathrm{pu}$ voir le jour. Ce qui veut dire que l'empreinte et les restes que laisse le corps mortel viennent avant la peinture, dans laquelle ils sont (re)transformés en images analogues, en copies de la face humaine, comparables à cette résurrection autour de laquelle 
gravite l'iconographie de la culture chrétienne. Ce processus touche fondamentalement aux origines de l'iconographie occidentale, ainsi que Hans Belting l'a démontré dans son essai sur les premières images du Christ, et qui porte le titre éloquent : «Face or Trace ${ }^{38} .^{38} \mathrm{Il}$ y thématise la genèse de l'iconographie chrétienne à partir du passage complexe entre les traces corporelles de la face du Christ sur le "suaire de Véronique " jusqu'à la représentation imagée de ce visage à partir des nombreuses peintures du Christ. Par la conversion du suaire, c'est-à-dire de la scène dans laquelle Véronique met sous les yeux de l'observateur en tant que témoignage 廆 le suaire avec les restes matériels 医, en l'image peinte d'un visage du Christ, a eu lieu la transformation de traces en une copie de la personne; transformation par laquelle se fonde la tradition imagée iconique qui a fondamentalement modelé le concept d'image occidental.

Cependant, l'histoire de l'image iconique et matérielle n'effleure qu'une partie de ce que désigne le concept d'image. Car, selon Mitchell: «Le vrai sens littéral du mot "image" réside dans une signification qui, de manière très décidée, ne recourt pas à des présentations imagées matérielles, qui s'érige plutôt contre elles. $"^{39}$ À la différence de Mitchell, je présume cependant qu'une compréhension de l'image qui ne réduit pas les images à des présentations visuelles ne doit en aucune façon se diriger nécessairement contre des images matérielles. Par opposition au conflit qui s'enflamme de manière répétée entre l'image et le texte - et de son écho dans les escarmouches entre les sciences du texte et les sciences de l'image -, ce qui m'intéresse c'est plutôt l'histoire de leur jeu conjugué et de leur interaction. Car le caractère imagé du langage et de la pensée ne doit pas nécessairement se trouver en opposition au visuel qui, sous forme de couleur, de toile, de photo, de film et d'autres médias adapte une forme aisthetique, c'est-à-dire sensible.

L'image et le texte ont depuis toujours été unis dans la tradition de l'ekphrasis (en grec: description), cet art de la description qui remonte à la rhétorique antique, et qui considère comme un idéal que le langage réussisse à présenter une scène « devant les yeux ». Dans la capacité de la littérature à dessiner avec des mots, et dans la faculté de l'art à raconter avec des images, le texte et l'image se donnent la main dans notre histoire de la culture..$^{40}$

Ce sont surtout les témoignages médiévaux qui attestent d'une autre forme d'interaction. Image et texte y sont les composantes d'égale valeur d'une écriture imagée, dont il n'est pas rare qu'elle apparaisse à des lecteurs contemporains comme une anticipation des comic strips - non pas seulement en raison des bulles qui communiquent les discours des figures représentées, mais aussi en raison de l'agencement des images comme narration, qui fait naître un récit imagé. ${ }^{41} \mathrm{Ce}$ n'est qu'avec la culture de l'ère Gutenberg que le texte et l'image eurent tendance à se séparer, et aussi parfois à s'affronter et à se combattre. Mais simultanément se formèrent aussi des genres particuliers à travers lesquels se poursuivit la tradition de l'iconicité de l'écriture ${ }^{42}$ et de l'écrit imagé. En témoigne toute la culture emblématique du Baroque, toutes ces formes de rhétorique, de théâtralité et d'écriture imagée auxquelles Walter Benjamin a consacré quelques-uns des chapitres les plus instructifs de son livre sur le drame baroque, particulièrement le passage sur «l'écriture stimulante » de l'allégorie, mais aussi les réflexions sur l'histoire de l'écriture, dans le fil de la doctrine de Johann Wilhelm Ritter : «Toute image n'est qu'une image écrite ».43 En témoignent aussi des genres artistiques comme les poèmes sur des tableaux, les poèmes de lettres et l'alphabet corporel. Ces genres profitent de la polysémie des 
caractères : comme désignation des lettres d'écriture et de personnes qui se distinguent par des qualités et des attitudes particulières. Et si, dans cette écriture d'images, les lettres de l'alphabet adoptent une forme physiognomonique ou anthropologique, cela réfère entre autres à l'origine picturale de certains systèmes alphabétiques, dans lesquels les caractères isolés ne sont pas seulement des lettres, mais représentent aussi des valeurs chiffrées ou des objets. Ainsi, dans l'alphabet hébreux, la lettre "beth » ne signifie pas seulement « $b$ » et le chiffre "deux", mais représente en même temps l'image et la désignation d'une maison.

C'est particulièrement à partir du chiffre « 0 » qu'a pu se développer un jeu productif avec les diverses fonctions qu'occupe ce seul et unique chiffre : simultanément l'omega et le zéro, la lettre et le signe numérique dans le code alphanumérique, et en outre, depuis l'introduction du 0 arabe dans le système de rédaction européen, signe de position qui a ouvert une culture à l'infinie multiplication : de un à dix, à cent et mille, il suffit à chaque fois d'ajouter un zéro. ${ }^{44}$ L'on ne saurait pas non plus envisager sans le « 0 » le système de notation des sciences modernes de la nature, dans lequel il figure, par exemple, le symbole de l'oxygène dans le système périodique de la chimie.

En même temps, de nombreuses associations imagées se lient à l'aspect visuel du « 0 » : depuis la pornographique Histoire d'O, jusqu'au jeu artistique avec la polysémie ellemême, comme on peut le voir par exemple dans la gravure de Jasper Johns: Color Numerals $\left(1969^{45}\right)$, en passant par l'image d'un foyer clos - qu'exploite la publicité d'un opérateur téléphonique allemand en montrant un couple qui s'est installé dans un « 0 » surdimensionné en s'y sentant pour ainsi dire " chez soi ", afin de connoter les services qu'elle offre par la simultanéité du foyer et du réseau global.

Les jeux avec la polysémie et la pluralité des formes de l'écriture ne se laissent pourtant pas seulement expérimenter sur les lettres et les textes, mais aussi sur des images, comme l'a montré Martin Kemp à l'exemple d'un « jeu de mots visuel », en plaçant la prise photographique d'une goutte d'eau par Arthur Worthington, A Study of splashes, à côté du logo de la firme "Milk Marque ». ${ }^{46}$ Dans ce jeu de mots visuel dans lequel l'image d'une goutte d'eau se transforme en symbole d'une couronne, l'image est entrée en mouvement. Ce qui revient à dire que dans l'art du jeu de mots et de significations également, la parenté entre le texte et l'image est plus grande que leur différence.

\section{Images actives}

Les images actives, que ce soit dans le langage ou dans le visuel, peuplent la scène des significations in actu - de ce côté-ci ou par-delà les significations fixées et codées, de ce côté-ci ou par-delà un royaume des signes -, à savoir là où les traces de couleurs, de lettres et de formes jouent leur propre jeu avec les analogies et les correspondances, et de la sorte, produisent des figures et des condensations inattendues.

Ce qui est en jeu par-delà le monde des signes, c'est de rouvrir, voire de briser des symboles et codifications figés, à l'instar de ces panneaux indicateurs dans lesquels la magie originelle des noms est non seulement sollicitée sous la forme de noms de rues servant de système citadin d'orientation et d'adresses, mais où ces noms, en plus d'indiquer le chemin, représentent l'archive d'un héritage national. C'est la raison pour laquelle les bouleversements politiques engendrent régulièrement des projets conflictuels de changements d'appellation des rues : une lutte pour maîtriser l'archive (nationale) de la mémoire culturelle dominante. L'installation de Raffael Rheinsberg, 
Gebrochen Deutsch [allemand brisé], montre un tel champ de bataille pour la conquête de la mémoire nationale: la surface de l'installation est composée de fragments de panneaux de rues de Berlin Est après leur nouvelle nomination depuis la chute du Mur. À partir de quelques combinaisons de lettres entières et écorchées, on peut reconstruire des mots isolés: "Dimitro» renvoie à rue Dimitroff, «fswald» à Greifswalder, «Prenzl » à Prenzlauer, et à plusieurs reprises il est possible d'identifier divers fragments des mots « allée » ou « rue ». Le succès du déchiffrement dépend de la connaissance du réseau des rues de Berlin Est. Plus on en est familier et plus d'infimes fragments suffisent à reconstruire d'autres noms de rues. Mais l'effet se produit aussi pour ceux qui connaissent moins bien les lieux; eux aussi se voient placés devant le champ de ruines d'une topographie symbolique qui renvoie à un événement du passé récent de la politique de la mémoire. En outre, le titre de l'installation ouvre sur une série d'associations qui se rapportent à la relation complexe entre langue et système de signes. ${ }^{47}$

Par-delà les concepts s'ouvre un champ dans lequel la littéralité de la langue fait remonter des significations oubliées qui sont au fondement de ces concepts, dont le sens a été aplani et abrasé par la familiarité et le naturel de l'usage quotidien - selon le mot célèbre de Karl Kraus : «Plus on regarde un mot de près, plus loin il regarde en arrière ». En effet, qui se souvient encore que le fondement de nos fondations découle $\mathrm{du}$ fond concret sur lequel nous nous tenons? Devant l'horizon de cet oubli quotidien des significations littérales, dû au caractère routinier et évident de la communication langagière, des modalités particulières d'écriture - par exemple, l'accentuation par l'utilisation de petites ou grandes capitales, italiques ou la séparation des composantes d'un mot - doivent rappeler que, dans nombre de mots sont cachées leurs significations tangibles et intuitives, exactement comme la lettre volée d'Edgar Allan Poe dans le récit éponyme : présente aux yeux de tous, personne ne la remarque. Par exemple, Ein-Sicht (voir dedans) [inspection], Ver-Antwort-ung (faire réponse) [responsabilité], Vor-läufige (qui court devant) [provisoire] et Voraus-gesetzte (qui est posé d'avance) [présupposé], ou encore ce Über-zeugen (témoigner/engendrer par-dessus/sur) [convaincre] à la polysémie duquel Walter Benjamin a consacré un magnifique aphorisme dans son Sens unique (1928) : « POUR HOMMES, Convaincre est infécond ». ${ }^{48}$ Le fait de convaincre [über zeugen - sur-témoigner/engendrer], le sur-accomplissement du témoin et du témoignage, le trop-faire, est une activité qui se prive elle-même de son résultat, tout comme la tentative de convaincre quelqu'un d'autre de quelque chose qu'il refuse d'admettre. ${ }^{49}$

En revanche, de ce côté-ci des concepts se trouve un vaste champ où sont avant tout chez elles les images langagières - en tant que métaphores qui précèdent les concepts formés. Ce sont là des images auprès desquelles nous nous réfugions lorsque nulle définition univoque n'est disponible, ou bien aussi des images à l'aide desquelles nous avançons en tâtonnant vers l'incertain, vers ce que nous ne savons pas précisément, et dans quoi, en dépit de tout, nous voulons ou devons opérer. Le philosophe Hans Blumenberg, dans sa théorie de l'inconceptuabilité, lui a donné le nom de «métaphore absolue $~_{5^{0}}$ À la différence des métaphores que l'on conçoit comme « façons de parler non spécifiques ", grâce auxquelles quelque chose d'étranger, d'inconnu ou de nouveau est reconduit à un registre familier; à la différence aussi de ces tropes maitres et métaphores dominantes de l'esprit du temps - comme programme, Mapping, information ou stockage -, à la différence donc de formules langagières disponibles, les métaphores absolues sont des images qui ne sont pas transposables en concepts : des 
mots du lexique d'un parler pré-spécifique. Les métaphores absolues sont des concepts in statu nascendi, lorsque la disponibilité d'une image permet d'approcher et de parcourir une portion de terre nouvelle. Blumenberg a développé le rôle de ces images à l'exemple de la «lisibilité de l'organisme »; une métaphore grâce à laquelle ont été déclenchées et forcées les recherches sur le code génétique, et qui ont finalement conduit au décryptage du génome humain. ${ }^{51}$ Avec l'achèvement de ce projet gigantesque, l'origine métaphorique du concept est cependant très largement tombée dans l'oubli.

C'est d'une opération métaphorique comparable que la nanotechnologie a reçu des impulsions décisives, comme l'a démontré l'informaticien Thomas Beth avec l'image du roulement à billes :

« Il incite à réfléchir au roulement à billes sur le plan moléculaire. [...] Ce n'est que plus tard que surgit la question de savoir si la formation doit être aussi idéale, en termes physico-mathématiques, qu'elle ressemble à un roulement à billes ou à un cristal bien propre. $\|^{52}$

À l'aide d'une métaphore, la faculté visuelle du langage en tant qu'expérience de pensée $^{53}$ se trouve activée, et ainsi en vient de temps en temps à générer des expériences de laboratoire et de nouvelles connaissances. Étant donné que l'image langagière relie des choses auparavant non liées, elle peut agir, dans la science, comme un éclair de connaissance auquel fait suite l'exploration systématique de champs inconnus - comme un "grondement longtemps après ", selon l'aphorisme connu de Walter Benjamin : «Dans les domaines qui nous occupent, il n'y a de connaissance que fulgurante. Le texte est le tonnerre qui fait entendre son grondement longtemps après. $1{ }^{54}$ Les domaines auxquels Benjamin fait ici allusion concernent l'histoire de la culture, mais dans les sciences de la nature par exemple, c'est l'expérience qui viendrait à prendre la place du texte. Certes, quiconque oublie que les roulements à billes moléculaires sont nés à partir d'un transfert depuis la mécanique vers l'organisme, se fermerait à la conscience que les métaphores représentent avant tout la possibilité de composer avec le caractère éternellement provisoire de nos connaissances. C'est devenu le projet de nombreux artistes que de rappeler ce caractère provisoire, en un temps où la science s'est appropriée toutes les possibilités des techniques visuelles.

Des installations comme Genesis, d'Eduardo Kac, opèrent sur le seuil entre la métaphore, le concept et les pratiques matérielles. Dans son installation, l'artiste a pris à la lettre la formule du "Livre de vie» en livrant une phrase de la Bible à l'expérience d'une traduction plurielle: de l'alphabet vers l'alphabet morse, puis vers l'alphabet de l'ADN, dont le résultat, lu comme une séquence, a fini par livrer le plan de construction pour la fabrication d'une bactérie. Après avoir modifié cette bactérie par des rayons ultraviolets, une retraduction en sens inverse fut entreprise : du code de l'ADN vers l'alphabet des lettres, en passant par l'alphabet morse. La modification s'exprimait au cours du processus par le déplacement partiel et la déformation de l'écriture. ${ }^{55}$ Les différences produites sur cette voie entre le résultat et la phrase de départ ont montré ceci : le statut de ce code n'est à comprendre ni de manière purement sémantique, en tant que patrimoine génétique, ni de manière purement mathématique, en tant qu'instruction pour la réplication. Il se présente plutôt comme système de rédaction d'une combinatoire dans laquelle réapparaît, par l'action conjuguée de lettres et de positions, cette écriture alphanumérique qui a précédé historiquement la séparation moderne entre lettres et chiffres. ${ }^{56}$ À ceci près que, maintenant, la combinatoire ne sert plus seulement à la présentation, à la disposition et à la répétition du savoir, mais 
qu'elle intervient en tant que praxis matérielle dans la structure moléculaire, autrement dit qu'elle re-combine la matière elle-même. Alors que bien des discussions scientifiques sur l'utilité et le contenu informatif du génome s'embrouillent, en raison du double sens du code génétique, entre écriture signifiante et algorithme, c'est à un projet artistique que revient le mérite d'avoir formulé ici l'une des réponses les plus claires. Le code de l'ADN représente un type d'écriture que nous pouvons certes manier, sur lequel nous pouvons aussi intervenir, sans en être toutefois ni l'auteur ni le créateur: un ars combinatoria qui - même sans être entièrement lisible - recèle de nombreuses possibilités de maniement.

Il en va de même avec la faculté poiétique et épistémique des images langagières et visuelles.

\section{BIBLIOGRAPHIE}

Bataille, Georges, «Lascaux ou la naissance de l'art », in : id., CEuvres complètes, tome IX, Paris, Gallimard, 1979.

Belting, Hans, Image et culte, traduit de l'allemand par Frank Muller, Paris, Éd. du Cerf, 1998.

Belting, Hans, « Face or Trace? Open question around the prehistory of Christ's Icon », in : Paleoslavica (Hommage à Ihor Sevcenko), Cambridge/Mass., 2003.

Benjamin, Walter, « Petite histoire de la photographie », in : id., Essais 1 (1922-1934), trad. Maurice de Gandillac, Éd. Denoël/Gonthier, 1983, p. 149-168.

Benjamin, Walter, Sens unique, traduit de l'allemand par Jean Lacoste, Paris, 10/18, 1978.

Benjamin, Walter, Origine du drame baroque allemand, trad. de l'allemand par Sybille Muller (avec le concours de André Hirt), Paris, Flammarion, 1985 [coll. Champs].

Benjamin, Walter, Le livre des passages, trad. par Jean Lacoste, Paris, Éd. du Cerf, 1989.

Benjamin, Walter, Paris, Capitale du XIX siècle, traduit de l'allemand par Jean Lacoste d'après l'édition originale de Rolf Tiedemann, Paris, Éd. du Cerf, 1989.

Benjamin, Walter, «L'œuvre d'art à l'époque de sa reproductibilité technique », in : Cuvres, vol. III, Paris, Gallimard, 2000.

Blumenberg, Hans, Paradigmes pour une métaphorologie, traduit de l'allemand par Didier Gamelin, postface de Jean-Claude Monod, Paris, Éd. Vrin, 2006 [coll. Problèmes \& Controverses].

Blumenberg, Hans, La lisibilité du monde, préfacé par Denis Trierweiler, traduction par Pierre Rusch et Denis Trierweiler, Paris, Éd. du Cerf, 2007.

Boehm, Gottfried / Pfotenhauer, Helmut (éd.), Beschreibungskunst - Kunstbeschreibung, Munich, 1995.

Bredekamp, Horst et al. (éd.), Theater der Natur und Kunst. Theatrum Naturae et Artis, Berlin, 2000.

Didi-Huberman, George, Devant l'image. Question posée aux fins d'une histoire de l'art, Paris, Minuit, 1990. 
Didi-Huberman, George, L'empreinte, Paris, Éd. du Centre Georges Pompidou, 1997.

Flusser, Vilem, «Die Auswanderung der Zahlen aus dem alphanumerischen Code », in : Matejovski, Dirk / Kittler, Friedrich (éd.), Literatur im Informationszeitalter, Francfort/Main / New York, 1996.

Göttert, Karl-Heinz, « Wider den toten Buchstaben. Zur Problemgeschichte eines topos », in : Kittler, Friedrich et al. (éd.), Zwischen Rauschen und Offenbarung. Zur Kultur- und Mediengeschichte der Stimme, Berlin, 2002, p. 93-113.

Graham, Dan, Ausgewählte Schriften, Stuttgart, 1994.

Gugerli, David / Orland, Barbara (éd.), Ganz normale Bilder. Historische Beiträge zur visuellen Herstellung von Selbstverständlichkeit, Zurich, 2000.

Hagner, Michael, Homo cerebralis. Der Wandel vom Seelenorgan zum Gehirn, Darmstadt, 1997.

Heine, Heinrich, « Die Reise von München nach Genua », in : id., Sämtliche Schriften, éd. par Klaus Briegleb, vol. 3, Munich, 1976.

Heinse, Wilhelm, Düsseldorfer Gemäldebriefe, éd. par Helmut Pfotenhauer, Francfort/Main / Leipzig, 1996.

Heintz, B. / Huber J. (éd.), Mit dem Auge denken. Strategien der Sichtbarmachung in wissenschaftlichen und virtuellen Welten, Zurich, 2001.

Ifrah, Georges, Universalgeschichte der Zahlen, Francfort/Main / New York, 1986.

Iglhaut, Stefan / Spring, Thomas (éd.), Science + fiction. Zwischen Nanowelt und globaler Kultur, Berlin, 2003.

Jones, Caroline A. / Galison, Peter (éd.), Picturing Science, Producing Art, New York / Londres, 1998. Johns, Jasper, Prints from the Leo Castelli Collection, Bruxelles, 1991.

Kac, Eduardo, Genesis, Maison européenne de la Photographie, Paris, 2002.

Kahn, Fritz, Das Leben des Menschen, 5 vol., Stuttgart, 1923-1931.

Kantorowicz, Ernst H., Les deux corps du roi, Paris, Gallimard, 1989.

Kemp, Martin, Bilderwissen. Die Anschaulichkeit naturwissenschaftlicher Phänomene, Cologne, 2003.

Kemp, Wolfgang(éd.), Theorie der Fotografie, I. 1839-1912, Munich, 1999.

Lipp, Carola (éd.), Medien popularer Kultur. Erzählung, Bild und Objekt in der volkskundlichen Forschung, Francfort/Main / New York, 1995.

Mitchell, W. J. T., « Was ist ein Bild ? », in : Bohn, Volker (éd.), Bildlichkeit. Internationale Beiträge zur Poetik, Francfort/Main, 1990.

Musil, Robert, « Denkmale », in : id., Gesammelte Werke, éd. par Adolf Frise, Reinbek près Hambourg, 1981.

Nauman, Bruce, Image / Text 1966-1996, Wolfsburg, 1997.

Rotman, Brian, Signifying Nothing. The Semiotics of Zero, Houndsmills / Basingstoke / Londres, 1987.

Schäffner, Wolfgang et al. (éd.), «Der liebe Gott steckt im Detail ». Mikrostrukturen des Wissens, Munich, 2003.

Schmitz, Ulrich / Enzel, Horst (éd.), Wissen und neue Medien. Bilder und Zeichen von 800 bis 2000, Berlin, 2003. 
Schwartz, Heinrich, David Octavius Hill. Der Meister der Photographie, avec 80 illustrations, Leipzig, 1931.

Stafford, Barbara, Kunstvolle Wissenschaft. Aufklärung, Unterhaltung und der Niedergang der visuellen Bildung, Dresde, 1998.

Weigel, Sigrid, Entstellte Ähnlichkeit. Walter Benjamins theoretische Schreibweise, Francfort/Main, 1997.

Weigel, Sigrid (éd.), Genealogie und Genetik. Schnittstellen zwischen Biologie und Kukturgeschichte, Berlin, 2002.

Weigel, Sigrid (éd.), Literatur als Voraussetzung der Kulturgeschichte. Schauplätze von Shakespeare bis Benjamin, Munich, 2004.

Weigel, Sigrid, « Das Gedankenexperiment - Nagelprobe auf die facultas fingendi in Wissenschaft und Literatur», in : Macho, Thomas / Wunschel, Annette (éd.), Science und Fiction. Über Gedankenexperimente in Wissenschaft, Philosophie und Literatur, Francfort/Main, 2004, p. 183-205.

Weigel, Sigrid, « Phantombilder zwischen messen und deuten. Bilder von Hirn und Gesicht in den Instrumentarien empirischer Forschung von Psychologie und Neurowissenschaft », in : Jagow, Bettina / Steger, Florian (éd.), Repräsentationen. Medizin und Ethik in Literatur und Kunst der Moderne, Heidelberg, 2004, p. 159-198.

Weigel, Sigrid, « Die innere Spannung im alphanumerischen Code (Flusser). Buchstabe und Zahl in grammatologischer und wissenschaftsgeschichtlicher Perspektive ", in : Dotzler, Bernhard / Weigel, Sigrid (éd.), « Fülle der Combination ». Literaturforschung und Wissenschaftsgeschichte, Munich, 2005, p. 357-380.

Wettengl, Kurt(éd.), Gedächtnis der Kunst. Geschichte und Erinnerung in der Kunst der Gegenwart, Francfort/Main, 2000.

Wundt, Wilhelm, « Lehre von den Gemüthsbewegungen », in : id., Philosophische Studien, Leipzig, 1891.

Zielinski, Siegfried, Archäologie der Medien. Zur Tiefenzeit des technischen Hörens und Sehens, Reinbek près Hambourg, 2002.

\section{NOTES}

1. Bien que la reproduction des images visuelles doive malheureusement faire défaut ici, je voudrais tout de même remercier Sabine Flach (Centre de recherches littéraires) pour son aide dans la recherche d'exemples appropriés.

2. Sur le temps des médias, cf. Siegfried Zielinski, Archäologie der Medien. Zur Tiefenzeit des technischen Hörens und Sehens, Reinbek près Hambourg, 2002.

3. Walter Benjamin, L'œuvre d'art à l'époque de sa reproductibilité technique, in : CEuvres, vol. III, Paris, Gallimard, 2000, p. 305.

4. Cf. par ex. Carola Lipp (éd.), Medien popularer Kultur. Erzählung, Bild und Objekt in der volkskundlichen Forschung, Francfort/Main / New York, 1995.

5. Martin Kemp, Bilderwissen. Die Anschaulichkeit naturwissenschaftlicher Phänomene, Cologne, 2003.

6. Comme la série de manifestations organisée par le Centre pour la recherche littéraire sous le titre WissensKünste, cf. Trajekte 3/2001 et 7/2003, ou bien l'exposition de l'Ars Electronica et l'exposition Science + fiction. Zwischen Nanowelt und globaler Kultur, éd. sur commande de la fondation Volkswagen par Stefan Iglhaut / Thomas Spring, Berlin, 2003. 
7. Cf. la reproduction in: B. Heintz / J. Huber (éd.), Mit dem Auge denken. Strategien der Sichtbarmachung in wissenschaftlichen und virtuellen Welten, Zurich, 2001, p. 118.

8. Heintz /Huber (2001), p. 147.

9. Heintz /Huber (2001), p. 73.

10. Cf. par exemple Barbara Stafford, Kunstvolle Wissenschaft. Aufklärung, Unterhaltung und der Niedergang der visuellen Bildung, Dresde, 1998. Caroline A. Jones / Peter Galison (éd.), Picturing Science, Producing Art, New York / Londres, 1998. Horst Bredekamp et al. (éd.), Theater der Natur und Kunst. Theatrum Naturae et Artis, Berlin, 2000. David Gugerli / Barbara Orland (éd.), Ganz normale Bilder. Historische Beiträge zur visuellen Herstellung von Selbstverständlichkeit, Zurich, 2000.

11. Cf. rep. in : Iglhaut / Spring (2003), p. 41.

12. Michael Hagner, Homo cerebralis. Der Wandel vom Seelenorgan zum Gehirn, Darmstadt, 1997, p. 99.

13. Fritz Kahn, Das Leben des Menschen, 5 vol., Stuttgart, 1923-1931.

14. Cf. rep. in : Iglhaut / Spring (2003), p. 229.

15. W. J. T. Mitchell, « Was ist ein Bild? », in : Bohn, Volker (éd.), Bildlichkeit. Internationale Beiträge zur Poetik, Francfort/Main, 1990, p. 21.

16. Wilhelm Wundt, «Lehre von den Gemüthsbewegungen », in: id., Philosophische Studien, Leipzig, 1891.

17. Cf. Sigrid Weigel, «Phantombilder zwischen messen und deuten. Bilder von Hirn und Gesicht in den Instrumentarien empirischer Forschung von Psychologie und Neurowissenschaft », in : Jagow, Bettina / Steger, Florian (éd.), Repräsentationen. Medizin und Ethik in Literatur und Kunst der Moderne, Heidelberg, 2004, p. 159-198.

18. Walter Benjamin, Le livre des passages, traduit de l'allemand par Jean Lacoste, Paris, Éd. du Cerf, 1989, p. 478.

19. Mitchell (1990), p. 21.

20. Cité d'après Iglhaut / Spring (2003), Textes et interviews, p. 15.

21. George Didi-Huberman, Devant l'image. Question posée aux fins d'une histoire de l'art, Paris, Minuit, 1990.

22. Hans Belting, Image et culte. Une histoire de l'art avant l'époque de l'art, traduit de l'allemand par Frank Muller, Paris, Éd. du Cerf, 1998.

23. Heinrich Heine, «Die Reise von München nach Genua », in : id., Sämtliche Schriften, éd. par Klaus Briegleb, vol. 3, Munich, 1976, p. 386 et 389.

24. Ernst H. Kantorowicz, Les deux corps du roi, Paris, Gallimard, 1989.

25. Robert Musil, "Denkmale », in : id., Gesammelte Werke, éd. par Adolf Frise, Reinbek près Hambourg, 1981, vol. 7, p. 505.

26. Cf. Karl-Heinz Göttert, "Wider den toten Buchstaben. Zur Problemgeschichte eines topos », in: Kittler, Friedrich et al. (éd.), Zwischen Rauschen und Offenbarung. Zur Kultur- und Mediengeschichte der Stimme, Berlin, 2002, p. 93-113.

27. Sur le rôle du détail pour la science de la culture, voir : Wolfgang Schäffner et al. (éd.), «Der liebe Gott steckt im Detail ». Mikrostrukturen des Wissens, Munich, 2003.

28. Ainsi John Leighton en 1853 lors d'une discussion à la " Photographic Society of London », in : Kemp, Wolfgang(éd.), Theorie der Fotografie I. 1839-1912, Munich, 1999, p. 91.

29. Wilhelm Heinse, Düsseldorfer Gemäldebriefe, éd. par Helmut Pfotenhauer, Francfort/Main / Leipzig, 1996, p. 69.

30. Cf. plus explicitement sur ce point Sigrid Weigel, « Die Richtung der Bilder. Zum Links-Rechts von Bilderzählung und Bildbeschreibung in kultur- und mediengeschichtlicher Perspektive », in : Weigel (2004a), p. 196-232.

31. Cf. par exemple Public Space / Two Audiences, 1976, in: Dan Graham, Ausgewählte Schriften, Stuttgart, 1994.

32. Cf. par exemple Dream Passage, 1983, in : Bruce Nauman, Image / Text 1966-1996, Wolfsburg, 1997. 
33. 5555 Meisterwerke, Directmedia, Berlin, 2000.

34. Walter Benjamin, «Petite histoire de la photographie ", in : id., Essais, 1922-1934, traduit de l'allemand par Maurice de Gandillac, Paris, Éd. Denoël/Gonthier, 1983, p. 154. La reproduction de la photographie se trouve dans Heinrich Schwartz, David Octavius Hill. Der Meister der Photographie. Avec 80 illustrations, Leipzig, 1931. Cf. aussi Sigrid Weigel, «Das Detail in Benjamins Theorie photo- und kinematographischer Bilder. Zur Verschränkung von Kultur- und Mediengeschichte », in : Weigel (2004a).

35. Keith Cottingham, "Fictitious Portraits. Studies for the Fictitious Portraits Series 1992 ", in : Amelunxen, Hubertus von (éd.), Photography after Photography. Memory and Representation in the Digital Age, Amsterdam, 1996.

36. Georges Bataille, «Lascaux ou la naissance de l'art », in : id., Cuvres complètes, tome IX, Paris, Gallimard, 1979.

37. Cf. Georges Didi-Huberman, L'empreinte, Paris, Éd. du Centre Georges Pompidou, 1997.

38. Selon Belting, l'image du Christ se distingue des types d'images connues dans le monde antique, parce qu'elle n'est ni l'image d'un corps mort, absent - comme c'est le cas dans les représentations funéraires traditionnelles - et ne peut pas non plus être rangée dans la série des images divines, de la présentation de divinités non visibles, surnaturelles, non corporelles. Bien plutôt l'image du Christ doit-elle se rapporter au statut énigmatique d'un corps certes mortel, mais ressuscité, dont il ne peut y avoir de présentation, tandis que les traces qui ont été laissées par le corps mort sont le seul témoignage. Dans cette mesure, le projet consistant à transformer ces traces en une image du visage du Christ est interprété par Belting comme énigme de l'image du Christ, par laquelle en viennent à s'exprimer les contradictions du christianisme lui-même. Voir Hans Belting, «Face or Trace? Open question around the Prehistory of Christ's Icon », in : Paleoslavica (Hommage à Ihor Sevcenko), Cambridge, Mass., 2003.

39. Mitchell (1990), p. 43.

40. Cf. Gottfried Boehm / Helmut Pfotenhauer (éd.), Beschreibungskunst - Kunstbeschreibung, Munich, 1995.

41. Cf. l'exemple d'une « Biblia pauperum» in : Ulrich Schmitz / Horst Wenzel (éd.), Wissen und neue Medien. Bilder und Zeichen von 800 bis 2000, Berlin, 2003, p. 86. Sur l'écriture imagée du MoyenÂge, cf. également l'exemple, discuté par Horst Wenzel, du « sermon à cinq doigts », ibid., p. 126.

42. Sur le concept de l'image écrite cf. Sybille Krämer, «Kann das geistige Auge sehen? Visualisierung und die Konstitution epistemischer Gegenstände », in : Heintz / Huber (2001), p. 350.

43. Walter Benjamin, Origine du drame baroque allemand, traduit de l'allemand par Sybille Muller (avec le concours de André Hirt), Paris, Flammarion, 1985 [coll. Champs], p. 231.

44. Georges Ifrah, Universalgeschichte der Zahlen, Francfort/Main / New York, 1986. Sur la sémiotique du zéro, cf. également Brian Rotman, Signifying Nothing. The Semiotics of Zero, Houndsmills / Basingstoke / Londres, 1987. Et sur la relation entre chiffres et lettres, cf. Sigrid Weigel, "Die innere Spannung im alphanumerischen Code (Flusser). Buchstabe und Zahl in grammatologischer und wissenschaftsgeschichtlicher Perspektive», in: Dotzler, Bernhard / Weigel, Sigrid (éd.), « fülle der combination ». Literaturforschung und Wissenschaftsgeschichte, Munich, 2005, p. 357-380.

45. Jasper Johns, Prints from the Leo Castelli Collection, Bruxelles, 1991.

46. Kemp (2003), p. 121 sq.

47. Cf. Kurt Wettengl(éd.), Gedächtnis der Kunst. Geschichte und Erinnerung in der Kunst der Gegenwart, Francfort/Main, 2000, p. 168.

48. « FÜR MÄNNER, Überzeugen ist unfruchtbar. » Cf. Walter Benjamin, Sens unique, traduit de l'allemand par Jean Lacoste, Paris, 10/18, 1978, p. 112.

N.d.T.: le lecteur aura compris que le caractère agglutinant, et transparent, de la langue 
allemande permet de retrouver le lexique le plus simple à partir duquel le miracle de la langue confectionne ensuite des significations complexes. D'où la nécessité ici de traduire mot à mot.

49. Cf. sur ce point le chap. VII in : Sigrid Weigel, Entstellte Ähnlichkeit. Walter Benjamins theoretische Schreibweise, Francfort/Main, 1997, p. 147 sq.

50. Hans Blumenberg, Paradigmes pour une métaphorologie, traduit de l'allemand par Didier Gamelin, postface de Jean-Claude Monod, Paris, éd. Vrin, 2006 [coll. Problèmes \& Controverses].

51. Hans Blumenberg, La lisibilité du monde, préfacé par Denis Trierweiler, traduit de l'allemand par Pierre Rusch et Denis Trierweiler, Paris, éd. du Cerf, 2007, p. 377-412 : « Le code génétique et ses lecteurs ». Cf. sur ce point : Sigrid Weigel, «Der Text der Genetik. Metaphorik als Symptom ungeklärter Probleme wissenschaftlicher Konzepte", in: id. (éd.), Genealogie und Genetik. Schnittstellen zwischen Biologie und Kukturgeschichte, Berlin, 2002.

52. Thomas Beth / Karlheinz Steinmüller, «Naturwissenschaft zwischen Publicity und Sciencefiction. Ein Gespräch mit Stefan Iglhaut und Thomas Spring », in : Iglhaut / Spring (2003), p. 237.

53. Cf. Sigrid Weigel, "Das Gedankenexperiment - Nagelprobe auf die facultas fingendi in Wissenschaft und Literatur», in : Macho, Thomas / Wunschel, Annette (éd.), Science und Fiction. Über Gedankenexperimente in Wissenschaft, Philosophie und Literatur, Francfort/Main, 2004, p. $183-205$.

54. Walter Benjamin, Paris, Capitale du XIX siècle, traduit de l'allemand par Jean Lacoste d'après l'édition originale de Rolf Tiedemann, Paris, Les éditions du Cerf, 1989, p. 473.

55. Eduardo Kac, Genesis, Maison européenne de la Photographie, Paris, 2002.

56. Cf. la contribution de Vilem Flusser, «Die Auswanderung der Zahlen aus dem alphanumerischen Code ", in: Matejovski, Dirk / Kittler, Friedrich (éd.), Literatur im Informationszeitalter, Francfort/Main / New York, 1996. Sur ce point, ma lecture de Flusser, note 44.

\section{INDEX}

Mots-clés : image, information, médias, texte

Schlüsselwörter : Bild, Information, Medien, Text

\section{AUTEURS}

\section{SIGRID WEIGEL}

Professeur de littérature, Université Technique, Berlin 